method consisted of shaking the gluten obtained from $5 \mathrm{gm}$. of flour with $100 \mathrm{c.c}$. of various concentrations of the two solvents for six hours at room temperature, filtering and analysing both the filtrate and solid for protein. The results given in the accompanying table show that 8 per cent sodium salicylate and 24 per cent urea are the minimum concentrations that will cause complete dispersion. Increasing the amount of solvent, or the time of extraction, had little effect on the amount dispersed.

The lower concentration of sodium salicylate required for dispersion, together with the fact that it does not contain nitrogen, favours this reagent over urea as a neutral solvent for gluten. The use of these neutral solvents for dispersing gluten is subject to criticism since they are known to denature other proteins, but it will be shown in papers to be published shortly in the Canadian Journal of Research that these reagents have a less drastic action on gluten than the classical solvents, dilute acid and alkali.

Division of Biology and Agriculture,

W. H. Cook.

National Research Laboratories, Ottawa, Canada.

Department of Field Crops,

R. C. Rose.

University of Alberta,

Edmonton, Canada.

1 Cook and Alsberg, Can. J. Res., 5, 355; 1931.

Cook, Can. J. Res., 5, 389; 1931.

\section{Shape of the Dibenzyl Molecule}

Some time ago, I completed an X-ray analysis of the crystal structure of dibenzyl ${ }^{1}$, and showed that the results could best be explained by a threedimensional model of the molecule, in which the planes of the benzene rings were at right angles to the plane containing the zig-zag of the connecting $\mathrm{CH}_{2}$ groups, as shown in Fig. 1. The structure is thus an interesting contrast to those aromatic compounds like naphthalene and durene in which all the carbon atoms of the molecule are found to lie in one plane.

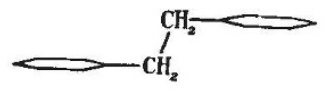

FIG. 1 .

More recently, however, Dhar ${ }^{2}$ has published the results of an independent investigation of dibenzyl, and his conclusions differ materially from mine. He assigns to the molecule the form shown in Fig. 2,

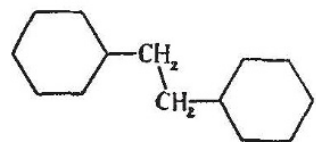

FIG. 2 .

with the modification that one benzene ring and its contiguous $\mathrm{CH}_{2}$ group are depressed below the plane of the paper by $0.12 \mathrm{~A}$. while the remainder is raised above the mean plane by a similar amount. His structure is thus only slightly distorted from the planar form.
To determine the exact shape of the molecule is obviously a matter of some difficulty, because the orientation with respect to the crystal axes is rather complicated. It is quite possible to place each of the above models in a position which makes the projections of the two structures in at least one direction very similar. The matter can only be settled by an exhaustive study of the reflections from several different zones in the erystal.

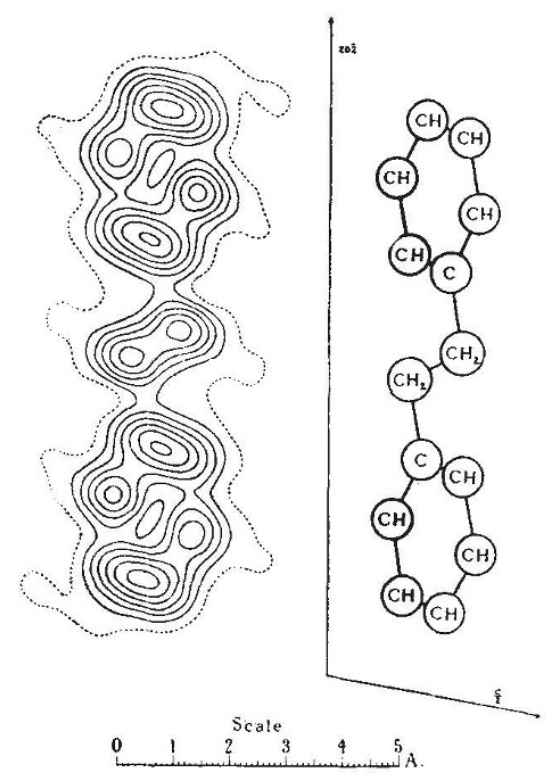

FIG. 3. Dibenzyl, projected along the $b$ axis. Fach contour line represents a density increment of one electron per square A.

I have now completed a double Fourier analysis of the structure factors for three such zones of reflections, and the results confirm my previous conclusions. The orientation given before is only slightly modified, and the shape of the molecule is verified. Calculations based on the Fourier analysis show that the planes of the benzene rings cannot be turned by more than about $8^{\circ}$ from the threedimensional position of Fig. 1.

A contoured map of the electron density for one of the projections (along the $b$ axis) is given in Fig. 3, and it will be seen that six of the fourteen carbon atoms are clearly resolved. The remainder are resolved in other projections, so that nearly all the co-ordinates can be estimated directly from these maps. The regularity of the benzene ring is a rather striking feature of this particular map. It was obtained by summing a double Fourier series of about fifty terms at 450 separate points taken over the asymmetric unit (half the molecule).

The possibility that Dhar has examined a different crystal modification from the one dealt with by me must not be overlooked. My crystals melted sharply at $52^{\circ}$, and corresponded in every respect with the standard description.

\section{J. Monteath Robertson.}

Davy Faraday Laboratory,

Royal Institution, London, W.1. July 27.

${ }^{1}$ Proc. Roy. Soc., A, 1934, in the press.

2 Current Science, 2, 480, June 1934. 